\title{
Adaptive cross-correlation detector for signals of optoelectronic reflective sensors
}

\author{
O.I. Bilyy ${ }^{1}$, R.Y. Yaremyk ${ }^{1}$, S.O. Kostyukevych ${ }^{2}$, K.V. Kostyukevych ${ }^{2}$ \\ ${ }^{1} L$ 'viv National University, Ukraine; \\ ${ }^{2} V$. Lashkaryov Institute of Semiconductor Physics, NAS of Ukraine, \\ 41, prospect Nauky, 03028 Kyiv, Ukraine
}

\begin{abstract}
A soft- and hardware realization of optoelectronic intellectual sensor for biomedical noninvasive studies based on the analysis of light reflected from living tissues has been described. The main feature of the developed model is use of an adaptive crosscorrelation detector controlled by the digital signal processor. Algorithms and operating mode of detector are defined by the type of particular problem to be solved and conditions of measurements. The proposed model was tested to identify dynamic signals in the following areas: pulsometer, evaluation variability of the cardial rhythm, evaluation of blood saturation by oxygen.
\end{abstract}

Keywords: optosensor, reflective sensor, cross-correlation detector, plethysmograph, pulsometry.

Manuscript received 16.01.09; accepted for publication 18.03.09; published online 20.03.09.

\section{Introduction}

When creating the optic-electronic sensory systems for biomedical noninvasive researches based on the analysis of light reflected from biotissues, one of the actual problems is neutralization of hindrances and artifacts that arise up in the process of measurements. The task to detect informative signals on a background of hindrances is especially sharply pronounced in the systems of monitoring in the conditions of the physical loadings, in the course of sportsman trainings, in rehabilitation process, in physical education etc. [1]. Experience of exploitation of optical noninvasive analyzers in clinical terms shows that, except for the traditional tasks of controlling the capacity of device and measuring terms, there arises a rather complicated problem to ascertain and perform numeral estimation of artifact levels that result in impermissible errors in device registration. Involuntary displacements of tissue in the operation area of touch-control lead to modulation of light beam pathlengths, which causes a change in the shape of the registered arterial pulsations, additional errors in mathematical treatment of informative signals corresponding to spectral components.

In the developed device, the methods of crosscorrelation analysis provide calculation of the ratio "useful signal/hindrance", the value of which is further utilized to estimate integrity of measurements and identification of errors. Inserted in the algorithm of device operation is additional a priori information about spectral composition of arterial pulsations and aperiodic noise hindrances. This approach is based on the experimentally fixed fact that pulsations of arterial blood are a practically periodic process within the frequency range $0.5-4 \mathrm{~Hz}$. On the other hand, the pulsations of hindrances, as a rule, have a random character with the central frequency $7 \mathrm{~Hz}$. Applying the cross-correlation function we have used the possibility to recover the lowpower periodic process in the case when a masking noise hindrance is aperiodic, i.e., it has a sufficiently wide spectrum and a respective quickly damped autocorrelation function. When the correlation interval is large enough, detected are only periodic components of the informative signal, while non-coherent noise hindrances are damped, as they are averaged in the cross-correlation integral [2].

\section{Structure and realization}

One of the principle lacks in construction of traditional measuring devices and systems is functional inflexibility inherent to the systems of the closed type, the parameters of which are set on the stage of development and are not optimized in the exploitation process. Therefore, when developing a functional diagram of intellectual optosensor detector of signals, we used the conception of 
open systems, characteristics of which are set not on the stage of design, but are operatively programmed and realized by user in the process of their exploitation in some optimum manner for the specific mode of measurements.

Creation of the open measuring system has that high-quality new maintenance that user gets a flexible instrument which can be operatively adapted to variable parameters of the process:

- to operatively configure the structure of the measuring system;

- to operatively change parameters of the structure: run-time range of measurements, period of digitization, bit capacity of analog-digital transformation;

- to reprogram the algorithm of system operation on the whole.

The functional diagram for realization of the intellectual detector of signals for optoelectronic reflective sensors is presented in Fig. 1.

The device is the mobile program-driven spectrophotometer to register the intensity of radiation with various wavelengths reflected from the probed biotissue, and the following treatment of data in time-domain and frequency-domain areas. As the sources of light beams, injection semiconductor laser emitters operating at three light wavelengths $\left(\lambda_{1}, \lambda_{2}, \lambda_{3}\right)$ of the spectral range are utilized in this setup. The law of light flux modulation for every source is realized on a program basis by using the method of current modulation in the $p-n$ junction via a digital-to-analog converter (DAC). The operation mode of emitters is pulsewise-periodic. Detection of the reflected informative signals is carried out by the optically screened photodetector matrix.
Every synchronous detector registers the increased photodetector signal only at the moment of arrival of the reference pulse synchronized with the beginning of the radiation pulse of the proper wavelength $\lambda$. At the synchronous detector output, the signal of $U_{s}(t, \lambda)$ is formed as proportional to the intensity of reflected radiation with this wavelength. The output signals of synchrodetectors are treated by analog-digital converters, the codes of measured values for every channel are added to the memory of controller for mathematical treatment. Application of a digital signal processor (DSP) allows to get the spectral parameters of measureable signals (Fourier-analysis, cross-correlation authentification) in a real-time mode. Graphic and text visualization of treatment results is provided by an internal liquid-crystal display (LCD), and at presence of connection with a host-computer data are written down in disk storage of the personal computer. The use of the personal computer allows to involve the archived databases and consulting model for synchronous work with a device. The service operation mode foresees programmatic reconfiguration of measuring structure and algorithms of data treatments, which provides an optimum in solving the specific tasks.

An increase in the signal/hindrance ratio after this cross-correlation treatment is caused by the fact that the autocorrelation function of noise hindrance in $\tau$-scale decays quickly, while the periodic components of the useful signal with growth $\tau$ appear periodically with the same intensity as at $\tau=0$. The greater time of integration, the more considerable improvement in the signal/hindrance ratio, as in this case averaging is realized on the greater amount of periods.

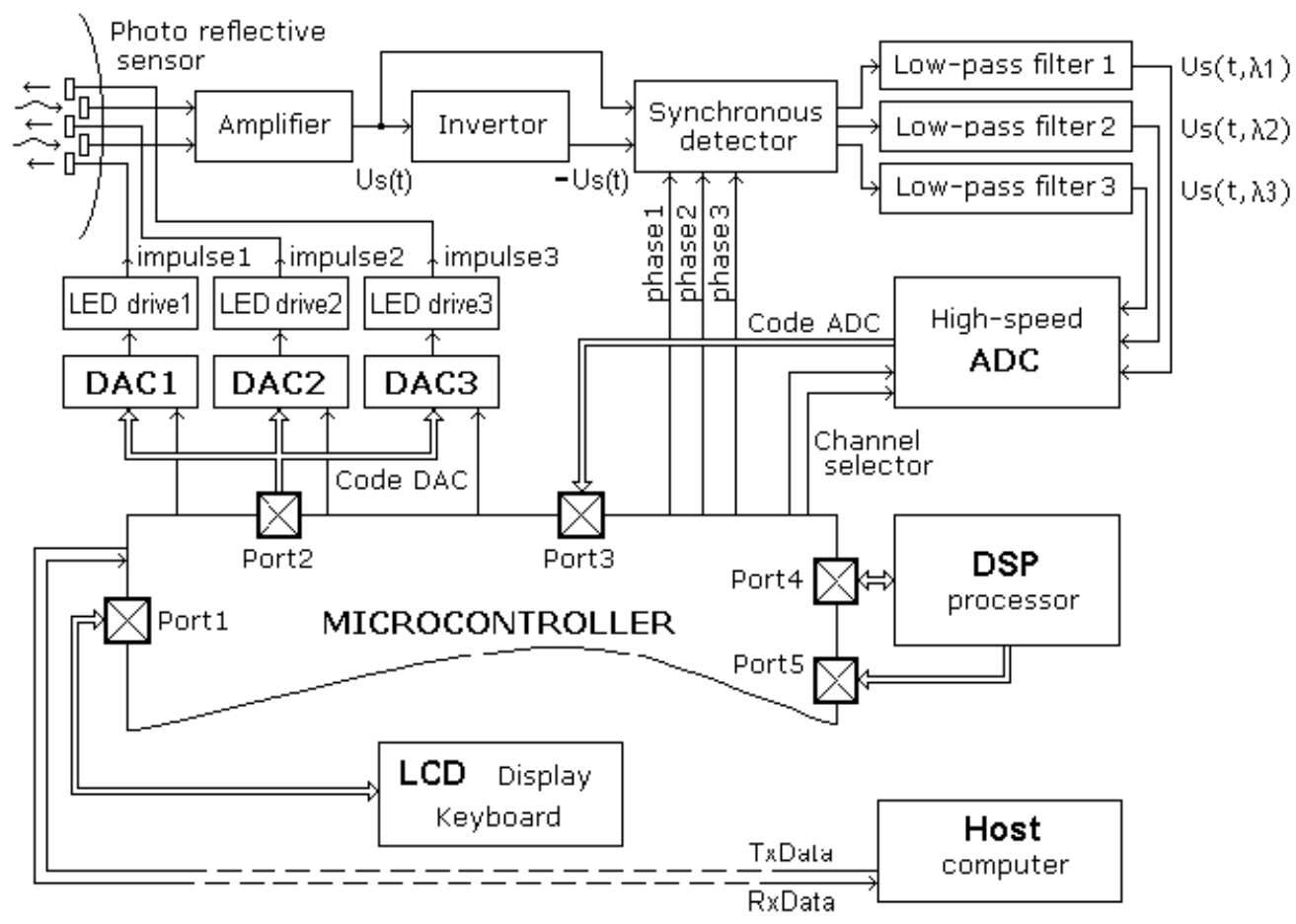

Fig. 1. Functional diagram for the cross-correlation detector of signals taken from optoelectronic reflective sensors. 
When $U_{s}(t)$ is the voltage of an informative signal, $U_{n}(t)$ is that of noise, the autocorrelation function of mixture will be determined by the following correlation:

$$
\begin{aligned}
& K(\tau)=\lim _{T \rightarrow \infty} \frac{1}{2 T} \times \\
& \times \int_{-\infty}^{+\infty}\left[U_{s}(t)+U_{n}(t)\right]\left[U_{s}(t+\tau)+U_{n}(t+\tau)\right] d \tau= \\
& =K_{s s}(\tau)+K_{n n}(\tau)+K_{s n}(\tau)+K_{n s}(\tau) .
\end{aligned}
$$

As the signal and noise are uncorrelated, $K_{s n}(\tau)=0$ and $K_{n s}(\tau)=0$. The autocorrelation function of noise $K_{n n}(\tau)$ at some $\tau$ value decays, too. This $\tau$ value depends on the top limit of noise band. So, as a hindrance in relation to the informative signal is aperiodic broadband process, there is a value $\tau=\tau_{\text {sup }}$, for which $K_{n n}(\tau)=0$ at $\tau>\tau_{\text {sup }}$, i.e., the relation $K(\tau)=K_{s s}(\tau)$ takes place. It means that after a sufficient time delay $\tau>\tau_{\text {sup }}$ the only autocorrelation function $K_{s s}$ of periodic informative signal remains.

The efficiency of autocorrelation method is limited by the small volume of information, which is comprised by the autocorrelation function. At a mutually crosscorrelation method, two signals are compared from different spectral channels, and, as a result of this crosscorrelation comparison, one gets very exact decisions that directly result in zero methods.

In this case, it is expedient to determine the function of intercorrelation, on the one hand between the mixture of useful signal and hindrances, and on the other hand - for the reconstructed signal based on a priori information. In this case, the autocorrelation function of hindrances $K_{n n}(\tau)$ is eliminated in principle. It can be represented by the following expression:

$$
\begin{aligned}
& K_{12}(\tau)=\lim _{T \rightarrow \infty} \frac{1}{2 T} \int_{-\infty}^{+\infty}\left[U_{s}(t)+U_{n}(t)\right]\left[U_{s}(t+\tau)\right] d \tau= \\
& =K_{s s}(\tau)+K_{s n}(\tau)=K_{s s}(\tau)
\end{aligned}
$$

The hindrance component $K_{s n}(\tau)$ disappears in the case when the signal and hindrance are uncorrelated and time of integration is sufficiently large. When studying periodic pulsations, the method of cross-correlation is of a great interest, so far as the useful signal of the expected periodicity can be selected by the cross-correlation method on the basis of comparing to the signal of the same frequency even in the conditions of strong hindrances that mask the informative signal $[2,3]$.

The principle of apparatus realization of the interface is based upon decentralized architecture of the program-managed analog-digital and digital-analog units, the majority of which are integrated into the chip of the multisignal controller produced by the firm Microchip. Control and synchronization of operation of the analog units are carried out by two highly-productive RISC-controllers connected by means of the standard communication port with the personal computer capable to reprogram flash-memory of the controllers depending on a specific task solved. It allows to realize the critical fragments of Assembler-language programs, to reach the maximum operation speed of the apparatus irrespective of the operation speed inherent to the used computer, to release the computer for performing another task.

Presence of the energy-independent program memory in the interface gives this module the opportunity of autonomous functioning with fulfilment of difficult measurements and calculating procedures (analog-digital signal conversion, analitical and statistical data processing) without displaying the results at the digital indicator or communicator.

The program part is an open system expanded by the user on the level of the cross-linker. The developed basic program module MPLIB is a complex of the interacting programs in the real-time scale that are implemented in Assembler for the architectures based on the PIC-controller by Microchip. The modularity and structurable nature of the program package secure transparency of the logical structure of all its elements.

We have also developed the program of graphic visualization of the experimental data and the results of their mathematical processing. This program allows automatic scaling and choosing different function by the user as arguments for comparative analysis.

\section{Conclusion}

Application of microelectronic technique of high integration degree and programmatic methods of adaptive cross-correlation treatment of measurement results is in three frequency-domain ranges and allows to substantially improve the functional parameters of the optoelectronic reflective sensor systems for noninvasive biomedical researches. Principles of construction of flow diagram, possibility of programmed control reconfiguration algorithm of device operation open prospects to realize a number of other tasks for noninvasive optical diagnostics.

\section{References}

1. O.I. Bilyy, K.O. Krapivina, B.G. Osinchuk, R.Y. Yaremyk, Intellectual photoplethysmographic sensor for sport medicine. Materials of the Third Congress of Ukrainian Biophysical Society with international participation, 2002, Lviv, Ukraine, p. 126.

2. F. Lange, Correlation Electronics. Gosudarstv. izdatel'stvo sudostroitel'noi promyshlennosti, Leningrad, 1983, p. 135 (in Russian).

3. S. Lawrence Marple, Jr. Digital Spectral Analysis with Applications. Mir Publ., Moscow, 1990, p. 257 (in Russian). 